\title{
Fabrication of Curcumin Encapsulated Chitosan-PVA Silver Nanocomposite Films for Improved Antimicrobial Activity
}

\author{
Kanikireddy Vimala ${ }^{1}$, Yallapu Murali Mohan ${ }^{1,2}$, Kokkarachedu Varaprasad ${ }^{1}$, Nagireddy Narayana \\ Redd $^{1}$, Sakey Ravindra ${ }^{1}$, Neppalli Sudhakar Naidu ${ }^{3}$, Konduru Mohana Raju ${ }^{1 *}$ \\ ${ }^{1}$ Synthetic Polymer Laboratory, Department of Polymer Science and Technology, Sri Krishnadevaraya University, Anantapur, India; \\ ${ }^{2}$ Cancer Biology Research Center, Sanford Research/USD, Sioux Falls, USA; ${ }^{3}$ Polymer Thin Film Laboratory, Department of \\ Chemical Engineering, Yonsei University, Seoul, South Korea. \\ Email: kmrmohan@yahoo.com
}

Received August 30 ${ }^{\text {th }}$, 2010; revised September 22 $2^{\text {th }}$, 2010; accepted September $28^{\text {th }}, 2010$.

\begin{abstract}
The present study explores the in situ fabrication of chitosan-poly(vinyl alcohol)-silver nanocomposite films in view of their increasing applications as antimicrobial packaging, wound dressing and antibacterial materials. The reduction of silver ions into silver nanoparticles (AgNPs) is achieved in acidic solution of chitosan $(C)$ and poly (vinyl alcohol) (PVA) using their functional groups (-OH, $-\mathrm{COOH},-\mathrm{NH}_{2}$ groups). The presence of silver nanoparticles in the chitosan-PVA film is confirmed by UV-Vis spectroscopy, Fourier Transform Infrared (FTIR) spectroscopy and X-ray Diffraction (XRD) analysis. The Scanning Electron Microscopic (SEM) images illustrate the presence of embedded silver nanoparticles throughout the films. In addition, the formed silver nanoparticles have an average particle size of $\sim 16.5$ $n m$ as observed by Transmission Electron Microscopy (TEM). The anti-microbial and anti-fungal activity of the chitosan-PVA silver nanoparticle films have demonstrated significant effects against Escherichia coli (E. coli), Pseudomonas, Staphylococcus, Micrococcus, Candida albicans, and Pseudomonas aeruginosa (P. aeruginosa). To improve further their therapeutic efficacy as anti-microbial agents, curcumin encapsulated chitosan-PVA silver nanocomposite films are developed which showed enormous growth inhibition of E. coli compared to curcumin and chitosan-PVA silver nanoparticles film alone. Therefore, the present study clearly provides novel antimicrobial films which are potentially useful in preventing/treating infections.
\end{abstract}

Keywords: Silver Nanoparticles, Chitosan, Poly(Vinyl Alcohol), Curcumin, Wound Dressing, Hydrogel

\section{Introduction}

Human beings are often infected by micro-organisms such as bacteria, yeast, mold, virus, etc [1]. Silver and silver ion based materials are widely known for their bactericidal and fungicidal activity. Their antimicrobial effect is due to blockage of respiratory enzyme pathways, alteration of microbial DNA and the cell wall [2]. Therefore, silver and silver ion containing materials are used as prostheses, catheters, vascular grafts and as wound dressings $[3,4]$. Recent studies have shown that silver in the form of nanoparticles is very effective as antimicrobial agent when compared to bulk silver or silver ions $[5,6]$. The therapeutic efficacy of silver in nanoparticles form is several folds greater than conventional silver compounds. The nanoparticles exert their antimicrobial property by interacting with the sulphur containing proteins present in bacterial cell membrane as well as with phosphorous containing DNA [6]. In addition, silver nanoparticles based antimicrobials have many advantages due to their thermal stability, health and environmental safety [7]. As a result, the usage of silver based commercial products including topical ointments, bandages, augmentation devices, tissue scaffolds, antimicrobial filters and gels have increased for improving public health care [8,9]. Some commercially available silver-containing purification systems such as Aqua pure, kinetico and QSI-Nano have shown to remove 99.99\% pathogens [10-13].

Many methods have been reported in developing silver nano-products including chemical reduction method. The chemical reduction method, using chemicals such as hy- 
drazine hydrate, dimethyl formamide, ethylene glycol, etc., however causes toxicity or biological hazards. The current trend in nanotechnology research is to use biocompatible or biological friendly polymers which can provide reduction as well as stabilization functions in the preparation of silver nanoparticles [14]. The combination of silver nanoparticles with water soluble biopolymers will produce new antimicrobials. Based on this, various natural polymers such as gum acacia, starch, gelatine, sodium alginate, carboxy methyl cellulose etc., have been employed to prepare biocompatible polymeric silver nanocomposites [15-17]. Chitosan, a natural polymer composed of poly( $\beta$-(1-4)-2-amino-2-deoxy-D-glucose), is one of the structural polysaccharide which is abundantly available in nature after cellulose. Chitosan interacts very easily with bacterium and binds to DNA, glycosaminoglycans and most of the proteins thereby enhancing the antimicrobial effect of silver nanoparticles [18]. Polyvinyl alcohol (PVA), a water soluble synthetic polymer, having less toxicity, possess excellent wound dressing bio reactor properties and known to be used as a stabilizer in nanoparticles synthesis $[19,20]$.

Films and coatings based on biopolymers function as barriers against moisture, oxygen, aroma flavor as well as oil and are the materials for future applications [21]. In addition, they have an interesting application as supports for antimicrobial, nutritional and antioxidant substances. Rira Jung et al. [11] prepared hydrated cellulose silver nanoparticle membranes and investigated their antimicrobial activity against $S$. aureus and E. coli. Durango et $a l$. [22] developed edible antimicrobial films using yarn starch and chitosan and shown to exhibit effectively the growth of S. enteritidis. Tripathi et al. [23] prepared chitosan-PVA blend films for food packaging applications and they have also shown to possess antimicrobial activity against food pathogenic bacteria.

In view of this, the present investigation involves the development of a novel, eco-friendly antimicrobial film containing chitosan, PVA and silver nanaoparticles by in situ fabrication method, as shown in Figure 1. The developed chitosan-PVA silver nanoparticles film was characterized with the help of UV-Vis, FTIR spectrophotometric, Thermogravimetric Analyzer (TGA), Scanning Electron Microscopy (SEM) and Transmission Electron Microscopy (TEM) analysis. It's antimicrobial activity against gram-positive (Staphylococcus and Micrococcus), gram-negative bacteria (E. coli and Pseudomonas), and fungi (Candida albicans and P. aeruginosa) has been tested. Curcumin (CUR), a hydrophobic polyphenolic compound derived from the rhizome of the herb curcuma longa, possesses a wide range of biological activities including wound healing, anti-bacterial, anti-oxidant, anti-inflammatory and anti-cancer properties.
Therefore this compound is incorporated into chitosan-PVA nanoparticles film to improve significantly the therapeutic antibacterial efficacy of the film.

\section{Experimental}

\subsection{Materials}

Chitosan (C) (high M.W, > 75\% deacetylated) is purchased from Sigma Chemical Company (St. Louis, USA). Acetic acid (glacial, 99-100\%), poly(vinyl alcohol) (PVA) (MW 125,000 and degree of acetylation, 19.5-22.7\%), silver nitrate $\left(\mathrm{AgNO}_{3}\right)$ and glutaraldehyde (GA) are purchased from Merck (Mumbai, India). Mineral salt broth and nutrient agar are obtained from Himedia Chemicals (Mumbai, India). The Department of Botany (Sri Krishnadevaraya University, Anantapur, India) has provided tandard cultures of the organisms. Curcumin $(95 \%(\mathrm{w} / \mathrm{w})$ curcuminoids by Spectrophotometry) is a gift sample from Natural Remedies Private Limited (Bangalore, India). All the chemicals and reagents are used without further purification. Double-distilled water is used for the preparation of all solutions throughout the study.

\subsection{Preparation of Chitosan-PVA Blend Films (CPB)}

$50 \mathrm{ml}$ of chitosan solution (1\% wt./wt. in acetic acid) and $50 \mathrm{ml}$ of PVA solution (1\% wt./wt. in water) (1:1) are mixed in $250 \mathrm{ml}$ beaker and stirred for $1 \mathrm{~h}$ at $60^{\circ} \mathrm{C}$ to obtain homogeneous solution. To this solution $1 \mathrm{ml}$ of $2 \%$ glutaraldehyde solution in water (a cross-linking agent) is added under stirring at room temperature $\left(25^{\circ} \mathrm{C}\right)$. The solution is transferred immediately into a Teflon sheet covered glass plate (Dimensions: $100 \mathrm{~mm}$ length $\mathrm{x}$ $100 \mathrm{~mm}$ width $\mathrm{x} 3 \mathrm{~mm}$ height) (Sabean traders, Chennai, India) and dried at $80^{\circ} \mathrm{C}$ in an electric oven for $2 \mathrm{~h}$ (Baheti Enterprises, Hyderabad, India). The formed cross-linked chitosan-PVA blend (CPB) films are washed with double distilled water to neutralization and dried at room temperature. This film is termed as 1:1 CPB film. Similarly, $50 \mathrm{ml}$ of $2 \%$ wt./wt. chitosan solution $+50 \mathrm{ml}$ of $1 \%$ wt./wt. PVA solution (2:1) and $50 \mathrm{ml}$ of $3 \% \mathrm{wt} . / \mathrm{wt}$. chitosan solution $+50 \mathrm{ml}$ of $1 \% \mathrm{wt} . / \mathrm{wt}$. PVA solution (3:1) are used to get different CPB films.

\subsection{Preparation of Chitosan-PVA Silver Nanoparticles Films (CPSNP)}

$\mathrm{AgNO}_{3}(100 \mathrm{mg})$ is added separately into three beakers containing $50 \mathrm{ml}$ of different percentages $(1 \%, 2 \%$ and $3 \%)$ of chitosan solutions at room temperature. The corresponding solutions are kept in sunlight for $1 \mathrm{~h}$. The yellow coloured solution started turning to red, then brown and brownish indicating the formation of silver nanoparticles. To this AgNP solutions, $50 \mathrm{ml}$ of 1\% PVA 
solution is added and stirrer for $1 \mathrm{~h}$. For all these solutions, $1 \mathrm{ml}$ of $2 \%$ glutaraldehyde (cross-linker) is added under stirring at room temperature. The solutions are then poured into Teflon covered glass plates and dried as explained earlier. These films are termed as chitosan-PVA silver nanoparticles (CPSNPs) films.

\subsection{Swelling Studies}

The completely dried, pre- weighed CPB and CPSNP films are equilibrated in $250 \mathrm{ml}$ of phosphate buffer $(\mathrm{pH}$ 7.4) at $25^{\circ} \mathrm{C}$. The water up take of the films is measured for every $30 \mathrm{~min}$ using analytical balance. The swelling ratio (Q) of the films is calculated using the following equation: $\mathrm{Q}=\mathrm{W}_{\mathrm{s}} / \mathrm{W}_{\mathrm{d}}$, where $\mathrm{Ws}$ is the weight of the swollen film at different time intervals and $W_{d}$ is the weight of dry film.

\subsection{Characterization}

The UV-vis spectroscopic studies are carried out using Shimadzu 1600 UV-vis spectrometer (Kyoto, Japan) 250 to $600 \mathrm{~nm}$. For this study, silver nanoparticles were extracted from silver nanocomposite film $(10 \mathrm{mg} / \mathrm{ml})$ over a period of a week, centrifuged at $1000 \mathrm{rpm}$ for $30 \mathrm{~min}$, and the supernatant was used to measure the absorption spectra. The distilled water was used as a blank solution. The UV spectra of the silver nanoparticles solution showed a characteristic peak between 413-421 nm related to surface Plasmon resonance absorption peak corresponding to silver nanoparticles. The FTIR spectra of CPB and CPSNP films are recorded on a MB3000 FT-IR Analyzer (ABB Analytical, Quebec, Canada).To record the FTIR spectra of films, the samples were completely dried in an oven(Baheti Enterprises, Hyderabad, India) at $40^{\circ} \mathrm{C}$ for $6 \mathrm{~h}$. These samples were read between 600 and $4000 \mathrm{~cm}^{-1}$ using $\mathrm{KBr}$ disk method. X-ray Diffraction (XRD) patterns were carried out for dried and finely grounded nanocomposite film samples on a Rigaku D/Max-2550Pc (Tokyo. Japan) using $\mathrm{Cu}$ and Ka radiation generated at $40 \mathrm{kV}$ and $50 \mathrm{~mA}$. Thermal studies of the films were carried out using SDT Q 600 TGA instrument (T.A. Instruments-water LLC, Newcastle, DE 19720, USA) at a heating rate of $10^{\circ} \mathrm{C} / \mathrm{min}$ under constant nitrogen flow $(100 \mathrm{ml} / \mathrm{min})$. Morphology of the films are observed by SEM through a JEOL JSM 840A (Tokyo, Japan).To image the film samples (surface or cross-sections) were coated with a thin layer of palladium gold alloy after mounting on a double sided carbon tape. TEM images are recorded using a Technai F 12 transmission electron microscope (Philips Electron Optics, Holland) operating at an acceleration voltage of 15 $\mathrm{kV}$. The samples are prepared for TEM measurements by extracting the silvernanoparticles from the film while they are in swollen stage using a soft ball and allowed to soak for 1 day to come out the silver nanoparticle from the film network into aqueous phase. From this solution 10-20 $\mu \mathrm{l}$ of the aqueous solution was dropped on a copper grid, removed the excess solution using filter paper and dried at room temperature. The copper grid was inserted into Technai F12 transmission electron microscope.

\subsection{Mechanical Properties}

Mechanical properties of CPB and CPSNP films are measured using a INSTRON 3369 Universal Testing Machine (UTM) (Buckinghamshire, England) running at a crosshead speed of $5 \mathrm{~mm} / \mathrm{min}$. The sample films are cut into $1 \mathrm{~cm} \times 10 \mathrm{~cm}$ size and the gauge length is about $5 \mathrm{~cm}$. The tensile parameters, maximum stress, young's modulus and \% elongation at break are measured using $10 \mathrm{~kg}$ load cell.

\subsection{Curcumin Loading and Encapsulation Efficiency}

Curcumin (CUR) is loaded into CPB or CPSNP films by swelling method. For loading curcumin, the films (50 mg) are allowed to swell in $20 \mathrm{ml}$ of CUR solution (5 $\mathrm{mg}$ of CUR in $20 \mathrm{ml}$, acetone (8 ml) - distilled water (12 ml)) for 24 hrs at $25^{\circ} \mathrm{C}$. The loading efficiency of curcumin in the films is determined spectrophotometrically [24]. The drug-loaded films are placed in $50 \mathrm{ml}$ of buffer solution and stirred vigorously for 96 hrs to extract the drug from the films. The solution is filtered and assayed by UV spectrophotometer at fixed $\lambda$ max value of $491.2 \mathrm{~nm}$. The results of \% drug loading and encapsulation efficiency are calculated using the following equations.

$\%$ Drug loading $=($ Weight of drug in film $/$ Weight of film) x 100

$\%$ Encapsulation efficiency = (\% actual loading / \% theoretical loading) x 100

\subsection{Release of Curcumin}

In order to study the release of curcumin from loaded films, known weights were placed in a measured volume (50ml) of $7.4 \mathrm{pH}$ phosphate buffer at room temperatures and the released amount of curcumin was determined at different time intervals by recording the absorbance of release medium by UV-Vis spectrophotometer. The recorded absorbance was then related to the amount of released curcumin using a calibration plot. The absorption of the solutions of curcumin was measured at $\lambda_{\max } 492.2$ $\mathrm{nm}$.

\subsection{Antimicrobial and Anti-fungal Activity}

The antimicrobial and antifungal activity of the developed CPB and CPSNP films are tested by disc diffusion, spread plate and viable cell count method against E. coli, 
Pseudomonas, Staphylococcus, Micrococcus, Candida albicans, and P. aeruginosa, as model bacteria and fungi.

For disc diffusion method, the films are cut into a disc shape with $5 \mathrm{~mm}$ diameter, sterilized by autoclaving for $30 \mathrm{~min}$ at $120^{\circ} \mathrm{C}$, and placed on different cultured agar plates. The plates are incubated for 2 days at $37^{\circ} \mathrm{C}$ in an incubation chamber maintaining with $5 \% \mathrm{CO}_{2}$ flow and the inhibition zone is then measured. Similarly, for spread plate method curcumin loaded CPB film and CPSNP films are placed in Petri plates. Then agar is poured onto the whole film and allowed to settle on the top of the film. The inhibition action of films is then measured. Viable count method measures the bacterial growth. For this study, $10^{8}$ colony forming units (CFU) of $E$. coli are grown in $10 \mathrm{ml}$ nutrient broth supplement with film discs (20 mm dia). The bacterial viability is checked using their O.D values by UV-Vis spectrometer at $600 \mathrm{~nm}$.

\section{Results and Discussion}

In the past few decades, a lot of work has been done and published on the development of synthetic and natural polymer AgNPs membranes/films as well as their action against various microorganisms $[24,25]$. However, these conventional films have exhibited limited applicability because of their low rate of fluid absorption and poor mechanical properties [26]. The films/membranes having AgNPs that are to be used for wound dressing and water purification purpose should posses good water absorption abilities and mechanical properties. Therefore, due to greater importance of chitosan and PVA in biomedical applications, chitosan-PVA silver nanoparticle films are developed via green process. Chitosan and PVA are well known polymers with excellent absorption capacities for a number of metal ions due to the presence of amino $\left(-\mathrm{NH}_{2}\right)$ and hydroxyl $(-\mathrm{OH})$ groups in their structures. The formation of the CPSNP is carried out by two step process as described in the experimental procedure (Figure 1).
In the first step, silver nitrate is added into the chitosan solution and the solution is irradiated by sunlight thereby reducing the silver ions into silver nanoparticles. In the second step, chitosan stabilized AgNPs solution is mixed with PVA solution and then glutaraldehyde cross-linker is added under stirring. The solution is allowed to form chitosan-PVA silver nanoparticles (CPSNPs) films. The AgNPs in these films are uniformly dispersed and entrapped throughout the networks via amine $\left(-\mathrm{NH}_{2}\right)$ and hydroxyl (-OH) functional groups [27,28]. Similarly curcumin loaded CPB and CPSNP films are developed by putting these films in curcumin solution. This approach is expected to improve the fluid absorption ability, mechanical strength as well as antimicrobial activities. Figure 2 represents the change in physical appearance of the films during the process of formation of silver nanoparticles and after loading the curcumin within the network. The light yellow colour film turned into dark brown colour due to the presence of Ag nanoparticle.

\subsection{Swelling Capacity}

The swelling capacity of an antibacterial film/gel and the nanocomposite plays an important role in the antibacterial activity, wound healing capacity, and for biomedical application due to their high water/solvent holding capacity. They can further absorb a slight to moderate amount of the wound exudates by swelling which helps in fast healing of the wound. Figure 3 shows the swelling capacity of CPB and CPSNP films with time. The CPB films showed higher swelling capacity than CPSNP films. For example, the swelling capacity of $1: 1 \mathrm{CPB}$ is $13.92 \mathrm{~g} / \mathrm{g}$ whereas the corresponding is CPSNP film is $9.8 \mathrm{~g} / \mathrm{g}$ only. This lowering in the swelling capacity is attributed due to binding of AgNPs with electrons of ' $\mathrm{O}$ ' and ' $\mathrm{N}$ ' atoms of hydroxyl and amine groups present in chitosan/PVA chains. This produces additional crosslink's within the chain networks [29]. The higher crosslink's within the films restrict the penetration of water for swelling [30,31].

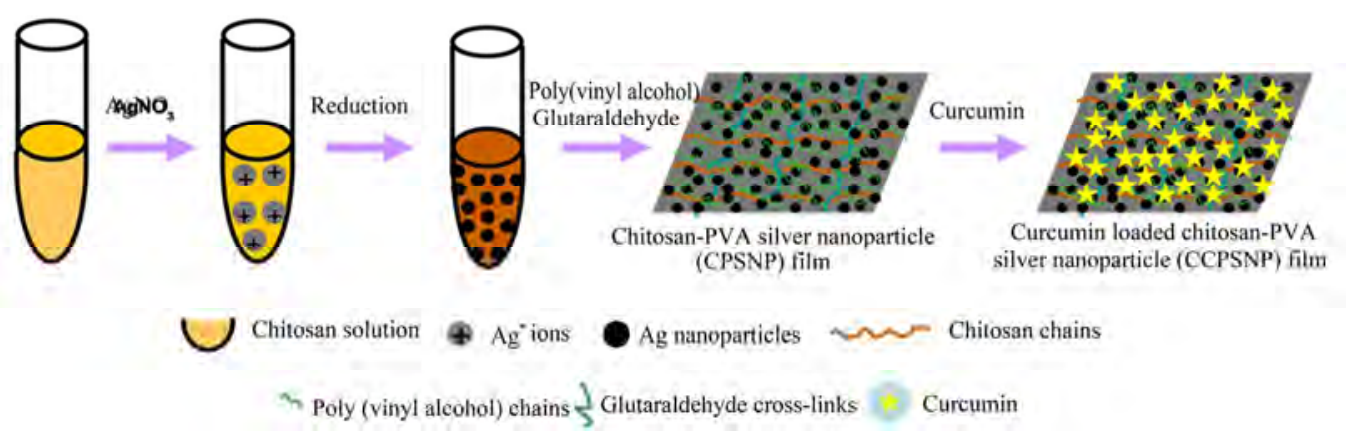

Figure 1. Schematic diagram of formation of chitosan-PVA silver nanoparticle films and curcumin loaded chitosan -PVA silver nanoparticle films. 


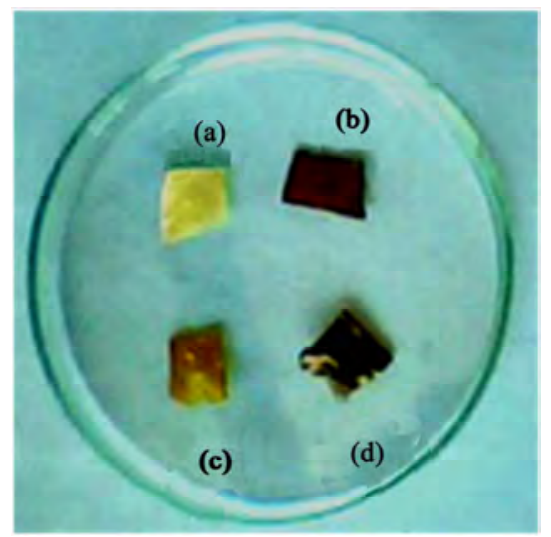

Figure 2. Representative (a) chitosan-PVA blend (CPB), (b) chitosan-PVA silver nanoparticle (CPSNP), (c) curcumin loaded chitosan-PVA blend, and (d) curcumin loaded chitosan-PVA silver nanoparticle films. These films were pre pared using 1:1 ratio of chitosan and PVA. Similar photo graphs were obtained for $2: 1$ and 3:1 ratio of chitosan and PVA.

The increase of chitosan content in the film (1:1, 2:1, 3:1, chitosan: PVA) increases the swelling capacity significantly i.e., 1:1 CPB $(13.92 \mathrm{~g} / \mathrm{g})<2: 1$ CPB $(16 \mathrm{~g} / \mathrm{g})<$ 3:1 CPB $(18.1 \mathrm{~g} / \mathrm{g})$. Similar trend is maintained in the case of CPSNP films i.e., 1:1 CPSNP $(9.8 \mathrm{~g} / \mathrm{g})<2: 1$ CPSNP (13.1)g/g < 3:1 CPSNP (16 g/g). This is due to the presence of more hydrophilic groups in the film networks which assist in improving the swelling characteristic of the films [32].

\subsection{UV-Vis Spectroscopy}

The formation of AgNPs is analyzed by UV-Vis spectroscopy. To evaluate the optimum composition of chitosan for the formation of silver nanoparticles, mild conditions are employed to avoid reducing agents. By increasing the concentration of chitosan from $1-3 \%(w / v)$ of films the intensity of surface plasmon resonance absorption peak corresponding to silver nanoparticles has grad- ually increased (0.105 to 0.195$)$ with a slight shift in the wavelength of the peak (413 to $421 \mathrm{~nm}$ ) (Figure 4).

The surface Plasmon resonance absorption peak is observed at 413-421 nm indicates the formation of silver nanoparticles of smaller size with narrow size distribution [33]. The reduction capacity as well as the stabilization of the formed nanoparticles increases with increase of chitosan concentration due to the presence more number of reducible groups [33]. The solution containing, $3 \%$ wt./v chitosan has shown an intense peak at $421 \mathrm{~nm}$ compared to other compositions. To study the effect of PVA in the formation of silver nanoparticles a film with the following composition consisting of chitosan (3\% wt./v.)-PVA (1\% wt./v.) solution is prepared. Surprisingly, the intensity of surface plasmon peak absorption has increased to 0.341 from 0.195 . This indicates the presence of PVA influences the reduction as well as the stabilization process of silver nanoparticles. From the foregoing experiments, we choose chitosan(3\%)

PVA(1\%) AgNP films for the forthcoming experiments. PVA, chitosan and CPB films have not shown any characteristic peak around $400-450 \mathrm{~nm}$ in the U.V. spectra (Data not shown).

\subsection{FTIR Spectra}

The FTIR spectra of CPB and CPSNP films are shown in Figure 5.

The CPB film (Figure 5a) has shown absorption peaks at $1664 \mathrm{~cm}^{-1}$ and $1325 \mathrm{~cm}^{-1}$ relating to amide I and III of $\mathrm{C}=\mathrm{O}$ stretching, $\mathrm{N}-\mathrm{H} / \mathrm{C}-\mathrm{N}$ stretching and $\mathrm{CH}_{2}$ wagging coupled with $\mathrm{OH}$ groups of chitosan respectively. The peak observed at $1454 \mathrm{~cm}^{-1}$ is due to $\mathrm{CH}_{2}$ bending, and the peak at $2933 \mathrm{~cm}^{-1}$ is characteristic of $-\mathrm{CH}_{2}$ asymmetric stretching of CS/PVA. The absorption peak observed at $3414 \mathrm{~cm}^{-1}$ indicates the hydrogen bonding nature of $\mathrm{OH} / \mathrm{NH}_{2}$ stretching. The silver nanoparticles loaded chitosan film (Figure 5b) has shown all the above characteristic peaks with a slight shift of the peak 1325 to
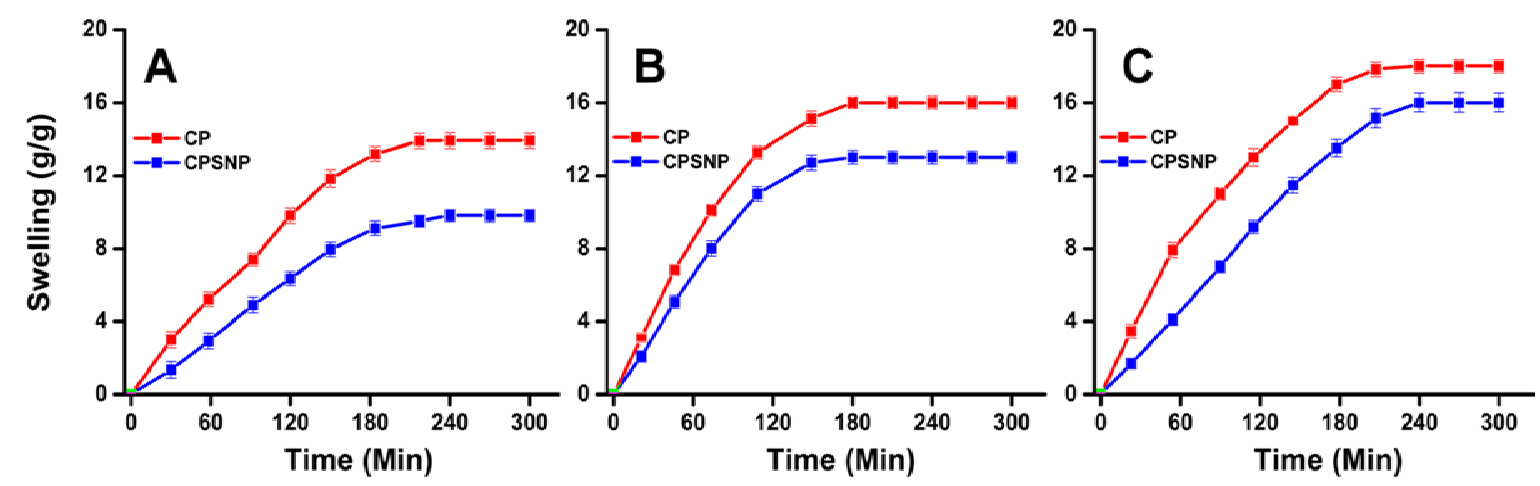

Figure 3. Swelling capacity of chitosan-PVA blend (CPB) and chitosan-PVA silver nanoparticle (CPSNP) films. (A) 1:1 (B) 2:1 and (C) 3:1 ratio of chitosan and PVA. 


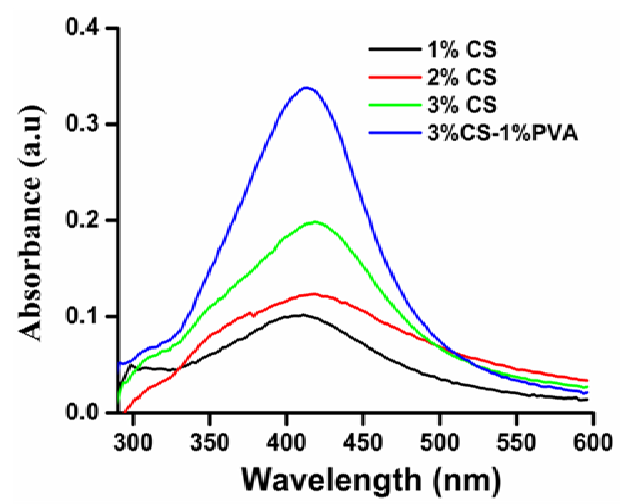

Figure 4. Surface plasmon resonance peak of silver nano particles generated using different concentrations of (1-3 wt./v.\% ) chitosan solutions and chitosan-PVA solutions.

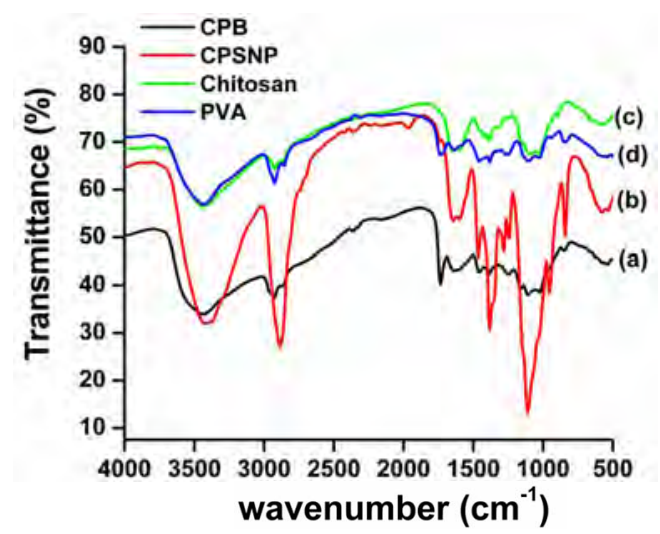

Figure 5. FTIR spectra of chitosan, PVA, CPB and CPSNP films.

$1352 \mathrm{~cm}^{-1}$ corresponding to amide III band. In addition, the stretching vibration at 3414 corresponding to $\mathrm{OH} / \mathrm{NH}_{2}$ groups has shifted to $3423 \mathrm{~cm}^{-1}$, indicating that the silver particles are bounded to the functional groups present both in chitosan and PVA. The shifting of the peak is due to formation of co-ordination bond between the silver atom and the electron rich groups (oxygen/nitrogen) present in chitosan. This causes an increase in bond length and frequency. Blank chitosan and PVA FTIR spectra are also depicted in Figure 5c and 5d for comparison purpose. All the above observations found in the IR spectra of films confirm the presence of silver nanoparticles in the chitosan-PVA film networks.

\subsection{XRD Analysis}

The X-ray Diffraction (XRD) is used to confirm the nature of crystal structure of the formed silver nano films (Figure 6A). Broad XRD patterns of CSB and CPSNP below $30^{\circ}$ (arrow mark) indicates the semi-crystalline nature of the major component i.e., chitosan. In addition, CPSNP has exhibited strong reflections around $38^{\circ}, 44^{\circ}$, $65^{\circ}$, and $78^{\circ}$ characteristic of (111) (200) (220) and (311) planes of face centered cubic (FCC) of the silver nanoparticles [33].

\subsection{Thermogravimetric Analysis}

Figure 6B illustrates the thermogravimetric analysis of CPB and CPSNP films. The initial weight loss (below $100^{\circ} \mathrm{C}$ ) observed in the films is due to loss of moisture present in the films. The weight loss observed in the case of CPB film is $80 \%$ around $490^{\circ} \mathrm{C}$ where as the weight loss observed for CPSNP at this temperature is only 73.83\%. The weight loss difference between the CPB and CPSNP represents the presents of silver nanoparticles in CPSNP. The percentage amount of silver nanoparticles present in the CPSNP film can be calculated from the difference in the weight loss between the CPB and CPSNP films at $800^{\circ} \mathrm{C}(\sim 6 \%)$, which is $\sim 5.57 \%$.

\subsection{Electron Microscopic Analysis}

The SEM analysis of the plain and silver nanoparticles loaded chitosan-PVA films are shown in Figure 7A. The plain CPB film (Figure 7A, a-b) has exhibited a dense and uniform plain microstructure. Whereas CPSNP film (Figure 7A, c-d) has shown the presence of defined nanoparticles in the film (arrows). To find out the exact size and morphology of the formed Ag nanoparticles in the CPSNP film, TEM analysis is done. The TEM image
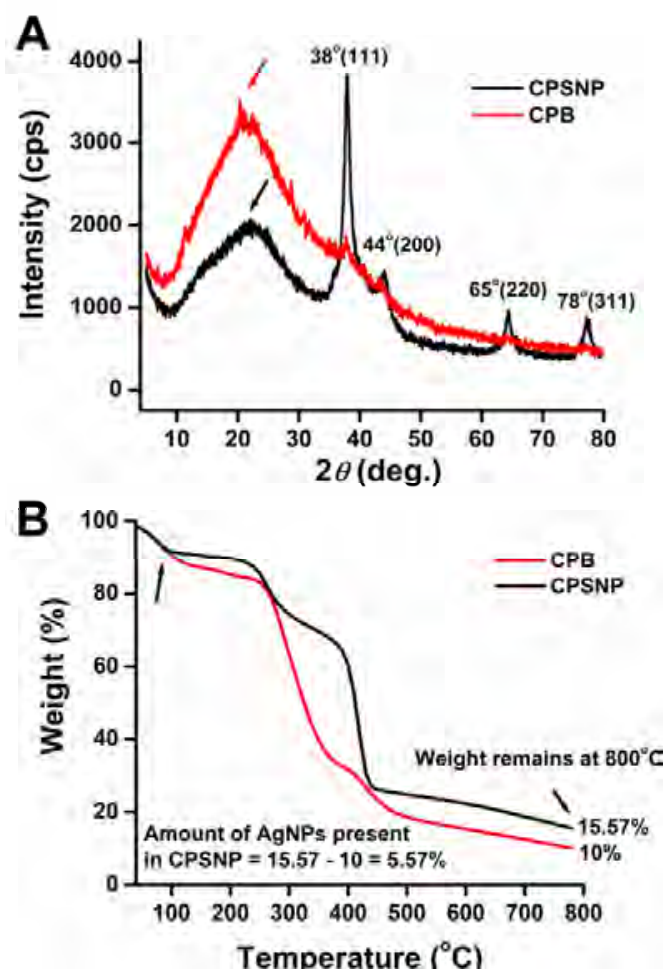

Figure 6. (A) X-ray diffraction patterns and (B) thermogravimetric analysis of CPB and CPSNP films. 
(a)
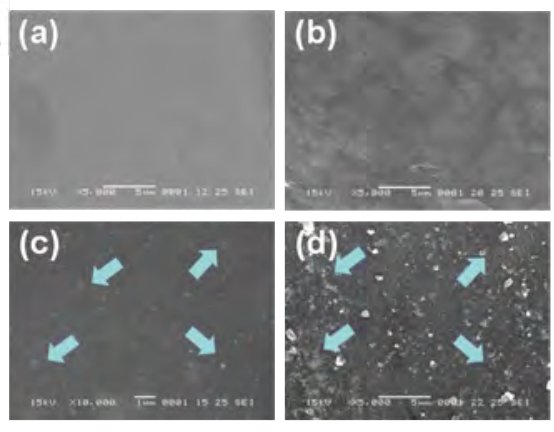

(b)

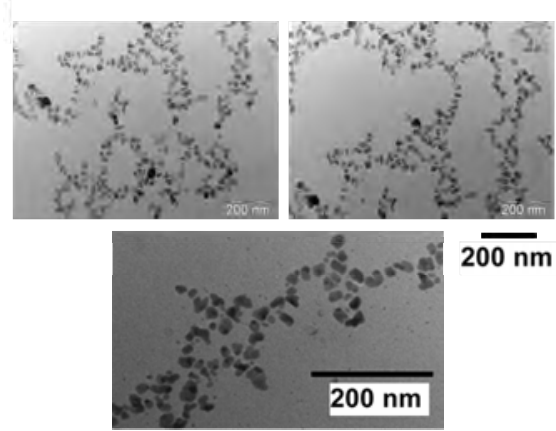

Figure 7. (A) Scanning electron microscopic images of (a-b) CPB and (c-d) CPSNP films. Arrows represent silver nanoparticles. (B) Transmission electron microscopy of silver nanoparticles obtained from CPSNP film.

of the silver nanoparticles extracted from CPSNP film is shown in Figure 7(b). The image indicates the particles are spherical in nature with dispersed morphology. The TEM results further illustrates that the particles formed have an average size of $\sim 16 \mathrm{~nm}$ (Figure 7(b)). As per the data available the silver nanoparticles generated in the chitosan-PVA film are essential for antimicrobial applications.

\subsection{Mechanical Properties}

Many synthetic and natural polymeric materials are developed to treat the burn wounds and as antibacterial materials. However, they have limited applicability due to poor mechanical properties as well as lower rates of water absorption. A number of chitosan- silver nanocomposites have been developed for many applications. To have better mechanical strength silver impregnated chitosan-PVA nanocomposites are developed in the present investigation for higher applicability. The mechanical properties of CPB and CPSNP films are presented in Table 1. The higher stress at maximum load, modulus, and elongation at break are noticed for CPSNP film compared to CPB film. The main objective of this investigation is to produce high strength to chitosan-PVA films and achieved by impregnating silver nanoparticles into Chitosan-PVA films.
Table 1. Mechanical properties of CPB and CPSNP films.

\begin{tabular}{cccc}
\hline $\begin{array}{c}\text { Sample } \\
\text { Code }\end{array}$ & $\begin{array}{c}\text { Stress at } \\
\text { Maximum } \\
\text { Load (MPa) }\end{array}$ & $\begin{array}{c}\text { Modulus } \\
(\mathrm{MPa})\end{array}$ & $\begin{array}{c}\text { Elongation at } \\
\text { break (\%) }\end{array}$ \\
\hline CPB & 26.6128 & 970.2833 & 4.492 \\
CPSNP & 33.274 & 1195.9382 & 5.467 \\
\hline
\end{tabular}

\subsection{In Vitro Release}

If these film materials are used as wound dressings, apart from absorbing wound exudates during the healing process, works as reservoirs to drugs for localized delivery to prevent bacterial infection. In the present study, curcumin (CUR) is selected as a model drug. Curcumin is the principal curcuminoid of the popular Indian spice turmeric, which is a member of the ginger family. It is used in ayurvedic and in Chinese medicine since long back [34]. In addition, it may be effective in treating malaria, prevention of cervical cancer, and may interface with the replication of HIV virus. Therefore loading efficiency of curcumin into films is examined (Table 2).

It is observed that the loading efficiency is higher in the case of CPSNP film compared to CPB films. This is due to absorption of more number of curcumin molecules on the silver nanoparticles in addition to entrapment in the films. Figures 8(a) and 8(b) gives the structure of curcumin and the drug delivery studies of $\mathrm{CPB}$ and CPSNP respectively.

To confirm whether the loaded curcumin in the films is in active form for functioning effectively in antibacterial application, the FT-IR analysis is performed. Figure 8(c) supports the presence of curcumin in both plain and Ag nanocomposites loaded films due to the presence of additional peaks at $1505 \mathrm{~cm}^{-1}$ and $1265 \mathrm{~cm}^{-1}$ corresponding to curcumin.

\subsection{Curcumin Release Kinetics}

The drug release kinetics was analyzed by plotting the cumulative release data verses time and by fitting the experimental release data into the following power law equation and determining the exponent,

$$
\mathrm{M}_{1} / \mathrm{M}_{\mathrm{eq}}=\mathrm{kt}^{\mathrm{n}}
$$

Where $M_{t}$ is the mass of water uptake at time $t, M_{e q}$ is the equilibrium water uptake, and $\mathrm{k}$ and $\mathrm{n}$ are constants, which are characteristic parameters of the specific (dis solution medium) system. A value of $n=0.5$ indicates the

Table 2. Loading efficiency of curcumin into CPB, CPSNP films, and curcumin release profiles from CPB and CPSNP films.

\begin{tabular}{ccccccc}
\hline Code & \%E & $\mathrm{n}$ & $\mathrm{k}\left(10^{2}\right)$ & $\mathrm{R}^{2}$ & $\begin{array}{c}\text { Release } \\
\mathrm{I}\end{array}$ & $\begin{array}{c}\text { Release } \\
\mathrm{II}\end{array}$ \\
\hline CPB & 69 & 0.99 & 0.58 & 0.98 & 12.87 & 100 \\
CPSNP & 81 & 1.27 & 1.47 & 0.98 & 6.26 & 97 \\
\hline
\end{tabular}



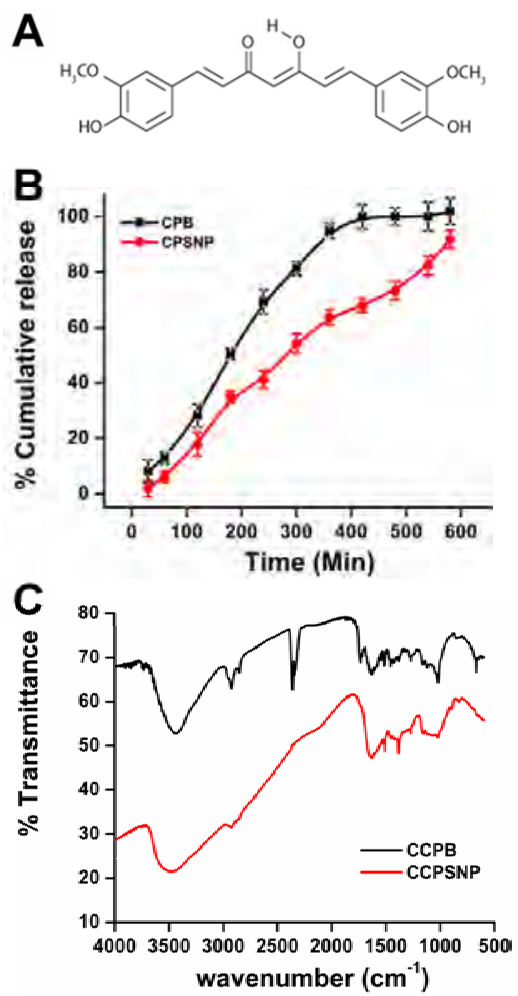

Figure 8. (A) Chemical structure of curcumin, (B) Curcumin release profile from CCPB and CCPSNP films, and (C) FTIR spectra of CCPB and CCPSNP films.

Fickian diffusion and $n=1$ implies case II transport; values of $\mathrm{n}$ between these limits define anomalous or non-Fickian transports. The values of ' $k$ ' and ' $n$ ' have been calculated by the least-squares method. These results along with correlation coefficients, $r$ values are presented in Table 2. The value of CCPB Films exponent ' $n$ ' is found to 0.99 as calculated from the empirical equation, respectively, which indicated anomalous nature. Similarly, the value of CCPSNP film exponent of $n$ is 1.27, which indicated that Zero order transport. The lower $\mathrm{k}$ values for all the systems indicate a lesser interaction between the film materials and the curcumin.

\subsection{Antimicrobial Activity}

The use of Ag-containing chitosan-PVA films as functional wound dressings is assessed by observing their antimicrobial activity (based on the disc diffusion method, spread plate method, viable cell count method) against some common bacteria and fungi found on burn wounds: E. coli, P. sudomonas, micrococcus, Staphylococcus, Candida albicus, and P. aeroginosa. Figure 9 exhibits the typical antimicrobial test results of films by the disc method. It is found that the CPSNP films have exhibited an inhibition zone (blue color arrows) whereas $\mathrm{CPB}$ film does not involve in the inhibition zone process

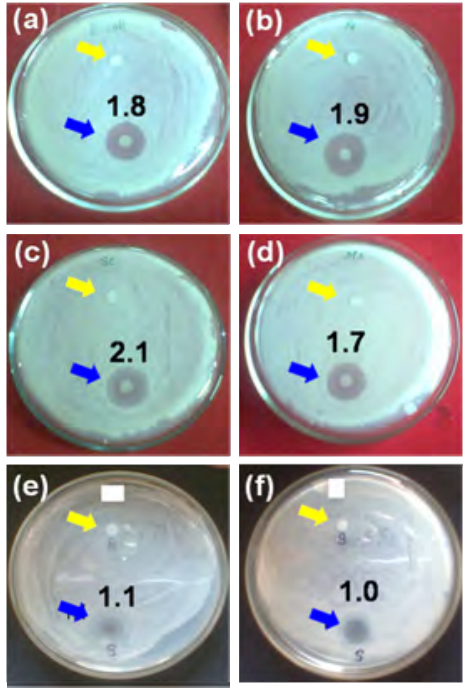

Figure 9. Anti-microbial and anti-fungal activity of CPB and CPSNP films against (a) E. coli (b) P. sudomonas (c) Staphylococcus (d) Micrococcus (e) Candida albicus (f) $P$. aeroginosa.

(yellow color arrows).

A second method is used to reveal the biocide action of curcumin loaded CPB and CPSNP films. The nutrient agar is spread into the Petri dish over the whole film and, the $E$. coli culture is streaked on the solid surface of the media. A significant difference is observed in the plates containing CCPB film and CCPSNP film (Figure 10). The observed results indicates that the CCPB film containing petridish has shown lesser bacterial colonies where as the CCPSNP film containing petridish exhibited almost no bacterial colonies. This clearly demonstrates that curcumin and nanoparticles loaded composite films have excellent bacterial inhibition growth capabilities.

The killing kinetics of the $E$. coli is also tested against CPB, CPSNP, CCPB and CCPSNP films by performing in mineral salt broth .The results suggest that a drastic bacterial growth inhibition is observed for a period of 7 hrs and the results are determined by O.D measurements (Figure 11). The order of the O.D values are CPB > CCPB > CPSNP > CCPSNP. The results clearly indicated that the CCPSNP film has exhibited excellent antimicrobial activity.

\section{Conclusion}

The present work demonstrates a simple method in producing novel chitosan-PVA silver nanocomposite films. The developed silver nanocomposite films have exhibited fairly good mechanical strength and superior antimicrobial properties. Further, the current work demonstrates a promising method to combine silver nano-composites with a natural compound (curcumin) in de- 

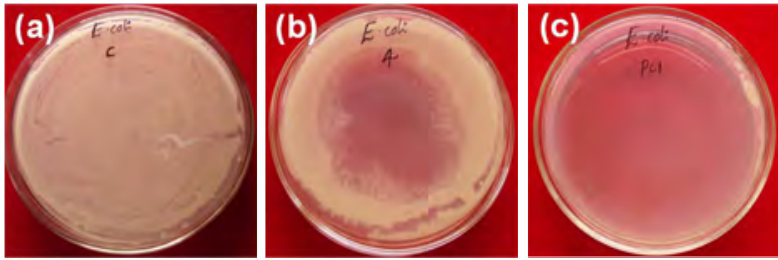

Figure 10. Antibacterial activity of (a) no treatment (control) (b) CCPB and (c) CCPSNP films.

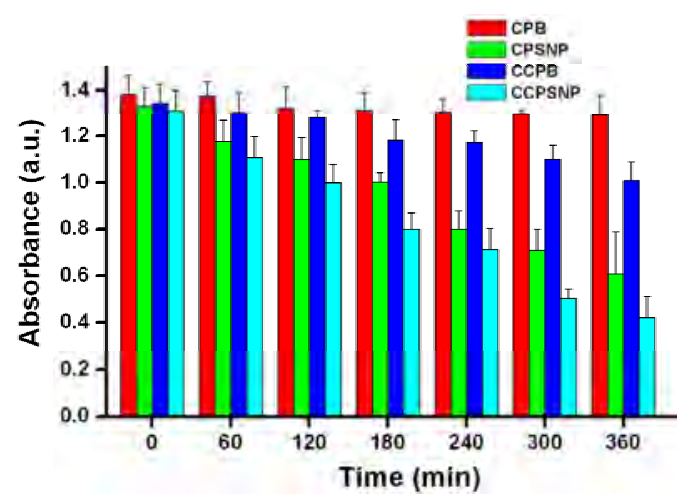

Figure 11. Antibacterial efficiency of CPB, CPSNP, CCPBand CCPSNP films against $E$. coli.

veloping novel antimicrobial agents .These agents may find potential applications in antimicrobial packaging materials and wound dressing/wound burns.

\section{Acknowledgements}

KMR thanks the Defence Research and Development Organization (DRDO) and Ministry of Defence, Government of India, New Delhi and KV thank U.G.C-SAP, New Delhi for the partial financial support.

\section{REFERENCES}

[1] D. G. Yu, M. Y. Teng, W. L. Chou, M. C. Yang, "Characterization and Inhibitory Effect of Antibacterial PAN Based Hollow Fiber Loaded with Silver Nitrate,” Journal of Membrane Science, Vol. 225, No. 1-2, November 2003, pp. 115-123. doi:10.1016/j.memsci.2003.08.010

[2] M. Rai, A.Yadav, A.Gade, "Silver Nanoparticles as A New Generation of Antimicrobial,” Biotechnology Advances, Vol. 27, No.1, January-February 2009, pp. 76-83. doi:10.1016/j.biotechadv.2008.09.002

[3] M. Catauro, M. G. Raucci, F. de Gaetano and A. Marotta, "Antibacterial and Bioactive Silver Containing $\mathrm{Na}_{2} \mathrm{O} \cdot \mathrm{CaO} \cdot 2 \mathrm{SiO}_{2}$ Glass Prepared by Sol Gel Method," Journal of Materials Science: Materials in Medicine, Vol.15, No.7, July 2004, pp. 831-837. doi:10.1023/B:JMSM.0000032825.51052.00

[4] J. H. Crabtree, R. J. Burchette, R. A. Siddiqi, I. T. Huen, L. L. Handott, A. Fishman, "The Efficacy of Silver-Ion Implanted Catheters in Reducing Peritoneal Dialysis-Related Infections, ” Peritoneal Dialysis International, Vol.
23, No.4, July- August 2003, pp.368-374.

[5] D.-C. Tien, K.-H. Tseng, C.-Y. Liao, T.-T. Tsung, "Identification and Quantification of Ionic Silver from Colloidal Silver Prepared by Electric Spark Discharge System and Its Antimicrobial Potency Study," Journal of Alloys and Compounds, Vol. 473, No. 1-2, April 2009, pp. 298-302. doi:10.1016/j.jallcom.2008.05.063

[6] J. S. Kim, E. Kuk, K. N.Yu, J.-H. Kim, S. J. Park, H. J Lee, S. H. Kim, Y. K. Park, Y. H. Park, C.-Y. Hwang, Y. K. Kim, Y.-S. Lee, D. H. Jeong, M.-H. Cho, “Antimicrobial Effects of Silver Nanoparticles," Nanomedicine: Nanotechnology, Biology and Medicine, Vol.3, No.1, March 2007, pp. 95-101.

doi:10.1016/j.nano.2006.12.001

[7] R. Kumar, H. Munstedt, "Polyamide/Silver Antimicrobials: Effect of Crystallinity on the Silver Ion Release," Polymer International, Vol. 54, No. 88, August 2005, pp. 1180-1186. doi:10.1002/pi.1828

[8] J. J. Castellano, S. M. Shafii, F.Ko, G. Donate, T. E. Wright, R. J. Mannari, W. G. Payne, D. J. Smith, M. C. Robson, "Comparative Evaluation of Silver Containing Antimicrobial Dressings and Drugs,” International Wound Journal, Vol. 4, No.2, June 2007, pp. 114-122. doi:10.1111/j.1742-481X.2007.00316.x

[9] M. Mishra, H. Kumar, K. Tripathi, "Diabetic Delayed Wound Healing and the Role of Silver Nanoparticles," Digest Journa of Nanomaterials and Biostrctures, Vol. 3, No. 2, June 2008, pp. 49-54.

[10] Q. Li, S. Mahendra, D. Y. Lyon, L. Brunet, M. V. Liga, D. Li, P. J. Alvarez, "Antimicrobial Nanomaterials for Water Disinfection and Microbial Control: Potential Applications and Implications,' 'Water Research, Vol. 42, No. 18, November 2008, pp. 4591-4602. doi:10.1016/j.watres.2008.08.015

[11] R. Jung, Y. Kim, H.-S. Kim, H. J. Jin, “Antimicrobial Properties of Hydrated Cellulose Membranes with Silver Nanoparticles,” Journal of Biomaterials Science: Polymer Edition, Vol. 20, No. 3, 2009, pp. 311-324. doi:10.1163/156856209X412182

[12] J. Jain, S. Arora, J. M. Rajwade, P. Omray, S. Khandelwal, K. M. Paknikar, "Silver Nanoparticles in Therapeutics: Development of an Antimicrobial Gel Formulation for Topical Use,” Molecular Pharmaceutics, Vol. 6, No. 5, May 2009, pp. 1388-1401. doi:10.1021/mp900056g

[13] A. Petica, S. Gavriliu, M. Lungu, N. Buruntea, C. Panzaru, "Colloidal Silver Solutions with Antimicrobial Properties,” Materials Science and Engineering: B. Vol. 152, No. 1-3, August 2008, pp. 22-27. doi:10.1016/j.mseb.2008.06.021

[14] S. K. Bajpai, Y.M. Mohan, M. Bajpai, R. Tankhiwale, V. Thomas, "Synthesis of Polymerstabilized Silver and Gold Nanostructures,” Journal of Nanoscience and Nanotechnology, Vol. 7, No. 9, September 2007, pp. 2994-3010. doi:10.1166/jnn.2007.911

[15] H. Kong, J. Jang, “Antibacterial Properties of Novel Poly(methyl methacrylate) Nano Fiber Containing Silver Nanoparticles,” Langmuir, Vol. 24, No.5, January 2008, pp. 2051-2056. doi:10.1021/la703085e 
[16] V. K. Sharma, R. A. Yongard, Y. Lin, "Silver Nanoparticles: Green Synthesis and Their Antimicrobial Activities," Advances in Colloid and Interface Science, Vol. 145, No. 1-2, January 2009, pp. 83-96. doi:10.1016/j.cis.2008.09.002

[17] V. Thomas, M. Namdeo, Y. Murali Mohan, S. K. Bajpai, M. Bajpai, "Review on Polymer Hydrogel and Microgel Metal Nanomposites: A Facile Nanotechnological Approach," Journal of Macromolecular Science, Part A: Pure Applied Chemistry, Vol. 45, No. 1, January 2008, pp. 107-119. doi:10.1080/10601320701683470

[18] M. Zeng, Z. Fang, C. X, "Novel Method of Preparing Microporous Membrane by Selective Dissolution of Chitosan/Poly Ethylene Glycol Blend Membrane,” Journal of Applied Polymer Science, Vol. 91, No. 5, March 2004, pp. 2840-2847. doi:10.1002/app.13469

[19] H-S. Kim, K-H. Lee, S-G. Kim, "Growth of Monodisperse Silver Nanoparticles in Polymer Matrix by Spray Pyrolysis,” Aerosol Science and Technology, Vol. 40, No. 7, July 2006, pp. 536-544. doi:10.1080/02786820600714361

[20] S. Liu, J. He, J. Xue, W. Ding, "Efficient Fabrication of Transparent Antimicrobial Poly (Vinyl Alcohol) Thin Films,” Journal Nanooparticle Research, Vol. 11, No.3, October 2007, pp. 553-560. doi:10.1007/s11051-007-9321-8

[21] S. Lu, W. Gao, H. Y. Gu,“Construction, Application and Biosafety of Silver Nanocrystalline Chitosan Wound Dressing," Burns, Vol. 34, No. 5, August 2008, pp. 623-628. doi:10.1016/j.burns.2007.08.020

[22] A. M. Durango, N. F. F. Soares, S. Benevides, J. Teixeira, M. Carvalho, C. Wobeto, N. J. Andrade, "Development and Evaluation of an Edible Antimicrobial Film Based on Yam Starch and Chitosan,” Packaging Technology Science, Vol. 19, No. 1, January-February 2006, pp. 55-59. doi:10.1002/pts.713

[23] S. Tripathia, G. K. Mehrotra and P. K. Dutta, "PhysicoChemical and Bioctivity of Crosslinked Chitosan-PVA Film for Food Packaging Applications,” International Journal of Biological Macromolecules, Vol. 45, No. 4, November 2009, pp. 372-376. doi:10.1016/j.ijbiomac.2009.07.006

[24] O. Suwantong, P. Opanasopit, U. Ruktanonchai, P. Supaphol, "Electrospun Cellulose Acetate Fiber Mats Containing Curcumin and Release Characteristic of the Herbal Substance,” Polymer, Vol. 48, No. 26, December 2007, pp. 7546-7557. doi:10.1016/j.polymer.2007.11.019

[25] S. K. Bajpai, Y. Murali Mohan, "Recent Advances in Nanoscience and Technology”, Bentham Science Publishers, Dubai, 2009.
[26] T. R. Thatiparti, A. Kano, A. Maruyama, A. Takahara, "Novel Silver-Loaded Semi-Interpenetrating Polymer Network Gel Films with Antibacterial Activity,” Journal of Polymer Science Part A: Polymer Chemistry, Vol. 47, No. 19, October 2009, pp.4950-4962. doi:10.1002/pola.23546

[27] A. Findon, G. McKay, H. S. Blair “Transport Studies for the Sorption of Copper Ions by Chitosan," Journal of Environmental Science and Health. Part A, Environmental science and engineering, Vol. 28, No. 11, 1993, pp. 173-185. doi:10.1080/10934529309375870

[28] N. G. Kandile, A. S. Nasr, "Environment Friendly Modified Chitosan Hydrogels as A Matrix for Adsorption of Metal Ions, Synthesis and Characterization,” Carbohydrate Polymers, Vol. 78, No. 4, November 2009, pp 753-759. doi:10.1016/j.carbpol.2009.06.008

[29] K. Vimala, K. Samba Sivudu, Y. Murali Mohan, B. Sreedhar, K. Mohana Raju, “Controlled Silver Nanoparticles Synthesis in Semi-Hydrogel Networks of Poly(Acrylamide) and Carbohydrates: A Rational Methodology for Antibacterial Application," Carbohydrate Polymers, Vol. 75, No. 4, February 2009, pp. 463-471. doi:10.1016/j.carbpol.2008.08.009

[30] K.Vimala, Y. Murali Mohan, K. Samba Sivudu, K. Varaprasad, S. Ravindra, N. Narayana Reddy, Y. Padma, B. Sreedhar, K. MohanaRaju, "Fabrication of Porous Chitosan Films Impregnated with Silver Nanoparticles: A Facile Approach for Superior Antibacterial Applications," Colloids and Surfaces B: Biointerfaces, Vol. 76, No. 1, March 2010, pp. 248-258.

doi:10.1016/j.colsurfb.2009.10.044

[31] J. Ostrowska-Czubenko, M. Gierszewska-Drużyńska, "Effect of Ionic Crosslinking on the Water State in Hydrogel Chitosan Membranes," Carbohydrate Polymers, Vol. 7, No. 3, July 2009, pp.590-598. doi:10.1016/j.carbpol.2009.01.036

[32] S. Ekici, D. Saraydin, "Interpenetrating Polymeric Network Hydrogels for Potential Gastrointestinal Drug Release,” Polymer International, Vol. 56, November 2007, No. 11, pp. 1371-1377.

[33] V. Thomas, Y. Murali Mohan, B. Sreedhar, S. K. Bajpai, "Fabrication, Characterization of Chitosan/Nanosilver Film and Its Potential Antibacterial Application,” Journal of Biomaterials Science, Part A: Polymer Edition, Vol. 20, No.14, 2009, pp. 2129-2144.

[34] H. Hatcher, R. Planalp, J. Cho, F. M. Torti, S. V. Torti, "Curcumin: From Ancient Medicine to Current Clinical Trials,” Cellular and Molecular Life Sciences, Vol. 65, No. 11, June 2008, pp. 1631-1652. doi:10.1007/s00018-008-7452-4 